\title{
Growth and characterization of ZnTe nanowires grown in a large scale by a CVD method
}

\author{
Mohammad Amin Baghchesara ${ }^{1}$, Mohsen Cheraghizade ${ }^{2}$, Ramin Yousefi $^{3 *}$ \\ ${ }^{1}$ Department of Metallurgy and Materials Engineering, Masjed-Soleiman Branch, Islamic Azad University (I.A.U), \\ Masjed-Soleiman, Iran \\ ${ }^{2}$ Department of Electrical Engineering, Bushehr Branch, Islamic Azad University (I.A.U.), Bushehr, Iran \\ ${ }^{3}$ Department of Physics, Masjed-Soleiman Branch, Islamic Azad University (I.A.U.), Masjed-Soleiman, Iran \\ *Corresponding author: E-mail: Yousefi.ramin@gmail.com, yousefiramin@iaumis.ac.ir
}

\begin{abstract}
ZnTe nanowires were grown in a big scale by tellurization of zinc sheets in a chemical vapor deposition (CVD) set-up. The zinc sheets were placed in three temperature zones in a tube furnace. Scanning electron microscope (SEM) images showed that, the ZnTe nanowires were grown with bigger diameter in the higher temperature. The phase and composition of the product were identified by X-ray diffraction (XRD) pattern and Xray photoelectron spectra (XPS). The XPS results showed that, the Te concentration was higher for the sample that was placed in the higher temperature. In addition, these nanowires produced a strong photoluminescence (PL) emission peak in the green region and a weak peak in the red region of the electromagnetic spectrum. Furthermore, a comparison study between the XPS and PL was carried out to understand origin of the defect emission of ZnTe nanowires.
\end{abstract}

Keywords: Crystal growth; Defects; Chemical vapor deposition; Semiconductors; Optical materials and properties.

\section{1- Introduction}

Recently research about synthesis and application of semiconducting metal chalcogenide nanocrystals is one of the hot topics [1]. Among metal chalcogenides, zinc telluride (ZnTe) is a semiconductor with a direct band gap of $2.26 \mathrm{eV}$ at room temperature and a Bohr exciton radius of $6.2 \mathrm{~nm}$, which is the best semiconductor for the green LED and photovoltaic applications [2-5]. Therefore, present a simple rout to grow ZnTe nanostructures such ZnTe nanowires could be led to develop optoelectronics devices in the future. Several methods such as molecular beam epitaxy (MBE) [6], hydrothermal [7], solvothermal [8], electrochemical [9], and metal-organic chemical vapor deposition (MOCVD) [10], which are usually complex, expensive, and time consuming, have been used to grow ZnTe nanostructures. On the other hand, the thermal evaporation method is the best method to grow ZnTe nanowires with high quality [11-13]. However, using the substrate as cation source can be changed this method to a cost effective method and growing the ZnTe nanowires in a big scale. 
Based on these reasons, we report and discuss the characterization of the ZnTe nanowires in a big scale prepared by a simple tellurization of a zinc sheet in a CVD set-up for the first time.

\section{2- Experimental}

The ZnTe nanowires were grown using the following procedure. Firstly, three high purity zinc sheets (99\%), with dimensions of $2 \times 2 \mathrm{~cm}$ and a thickness of $1 \mathrm{~mm}$ were used as substrates. The sheets were cleaned by an electroEtching method. Then the sheets were put into a horizontal tube furnace (CVD set-up) at 345, 375, and 405 ${ }^{\circ} \mathrm{C}$. Stoichiometric tellurium powder (Sigma-Alderich, tellurium (Te) $(99.99 \%)$ ) was used as the source material to create the tellurium ambiance in the tube furnace, in an alumina boat at $450{ }^{\circ} \mathrm{C}$. A high purity $\operatorname{Ar}(99 \%) / \mathrm{H}_{2}(10 \%)$ gas was fed into the furnace as carrier gas at about $120 \mathrm{sccm}$ at one end, while the other end was connected to a rotary pump. The growth process was allowed to proceed for $1 \mathrm{~h}$. A vacuum of 50 Torr was maintained inside the tube furnace.

The morphology, crystal structure, and elemental contents of the products were investigated using a scanning electron microscope (SEM; Hitachi S4160), an X-ray diffractometer (XRD; Siefert ID 3003), energy dispersive xray (EDX, Quanta $200 \mathrm{~F}$ ) and x-ray photoelectron spectra (XPS) (VG-Microtech ESCA-2000 with $\mathrm{Mg}_{\mathrm{K} \alpha}$ radiation as the excitation source), respectively. Room temperature photoluminescence (Jobin Yvon Horiba HR 800 UV), was employed to study the optical properties of the ZnTe nanowires. A He-Cd laser with $325 \mathrm{~nm}$ emission was applied for the PL excitation.

\section{3- Results and discussion}

Figure 1 shows SEM images of the ZnTe nanowires that are grown on all zinc sheets. Figure 1 (a-c) shows the FESEM images with different magnification of ZnTe nanowires that are grown in the low temperature zone. The average diameter of the nanowires in this area is around $30 \mathrm{~nm}$. On the other hand, the nanowires, which are grown in the middle (Fig. 1(d-f)) and high (Fig. 1(g-i)) temperature zones, have bigger diameters. In addition, the shape of the nanowires is different. Probably, it can be discussed such results by the EDX results. It can be seen, tellurium content is different in the different zones. In fact, the nanowires, which are grown in the higher temperature zone, have higher tellurium content. Usually, anion concentration is the most important factor to obtain different

morphology in metal-chalcogenide nanostructures [14]. Therefore, according to temperature role as a factor to control tellurium concentration in the set-up, obtaining different morphology could be due to different tellurium 
concentration. In addition, the EDX spectra of the samples show oxygen peaks that could be due to leakage of the set-up.

Figure 2 shows the XRD patterns of the samples. The XRD patterns in Fig. 2 agree with the standard card of bulk ZnTe (JCPDS Card No. 15-0746), which indicates the Zincblend structure. The addition of the ZnTe peaks; the zinc peaks from the sheets are also detected. However, the zinc peaks are weaker by increasing temperature.

Figure 3 is the high-resolution XPS spectra of the ZnTe nanowires that were formed in the different temperature. As can be seen, the spin-orbit splitting of $23 \mathrm{eV}$ for $\mathrm{Zn}-2 \mathrm{p}_{3 / 2}$ and $\mathrm{Zn}-2 \mathrm{p}_{1 / 2}$ confirms that the $\mathrm{Zn}$ atoms are in a ZnTe lattice [15]. In the case of Te, there appear two peaks at 573 and $583.3 \mathrm{eV}$, which corresponds to $3 \mathrm{~d}_{5 / 2}$ and $3 \mathrm{~d}_{3 / 2}$ transitions, respectively. In addition, in adjacent to these two peaks there appears another set of peaks at 576.6 and $587 \mathrm{eV}$, which corresponds to $3 \mathrm{~d}_{5 / 2}$ and $3 \mathrm{~d}_{3 / 2}$ transitions from the oxide phase of Te (Te-O) [15]. It can be seen the oxidation state is decreased in the higher temperature. Therefore, the nanowires that are grown in the higher temperature have closer structure to the $\mathrm{ZnTe}$ structure in comparison to the nanowires structure that are grown in the lower temperature. In fact, higher temperature zone is supersaturation zone of Te. Therefore, higher temperature zone is tellurium rich zone, this claim can be proven by EDX results and surface area under Te peaks in the XPS spectra.

Figure 4 shows the room temperature PL spectra of the ZnTe nanowires. There are two bands in the PL spectra of the products, one of them related to the green emission at $534-542 \mathrm{~nm}(2.28-2.32 \mathrm{eV})$ and the other related to the red emission at 656-659 $\mathrm{nm}$. The green emission is about to the near band edge (NBE) emission, due to the recombination of free excitons through an exciton-exciton collision process [7]. The red emission could be due to defects such as Te vacancy. In fact, according to the EDX and XPS results, the nanowires, which were grown in the lower temperature, have lower Te concentration in comparison to the nanowires that were grown in the higher temperature. Nishio et al. reported that $\mathrm{Te} / \mathrm{Zn}$ ratio in the $\mathrm{ZnTe}$ structure is an important factor to obtain different feature of the PL spectrum of ZnTe [16]. They showed that, small ratio cause to appear the red emission in the PL spectrum of ZnTe thin film. Furthermore, Chen et al. suggested that, such emission is due to $\mathrm{O}_{\mathrm{Te}}$ emission as a defect in the ZnTe lattice [17]. In fact, the ZnTe nanowires, which were grown in the lower temperature, have smaller Te/Zn ratio and more Te-O banding according to the EDX and XPS results.

\section{4- Conclusion}


A simple tellurization of the zinc sheets, which were placed at different temperatures, in a CVD set-up was used to grow ZnTe nanowires. The SEM images showed that, the morphology of the ZnTe nanowires depended on the temperature strongly. According to the EDX and XPS results, Te concentration was the most important factor to obtain different morphologies of the ZnTe nanowires. The PL results of the ZnTe nanowires showed that the band gap of the ZnTe nanowires was in the green region of the electromagnetic spectrum. In addition, the PL results showed that Te vacancy and Te-O bonding were two factors that caused to appear red emission in the PL spectra of the ZnTe nanowires.

\section{Acknowledgment}

M.A. Baghchesara gratefully acknowledges obtaining a research grant from Iran National Science Foundation (INSF). In addition, M.A. Baghchesara and R. Yousefi acknowledge the Islamic Azad University (I.A.U), Masjed-Soleiman Branch, for its technical support of this research work.

\section{References}

[1] Yousefi R, Jamali-Sheini F, Khorsand Zak A. Metal chalcogenide hierarchical nanostructures for energy conversion devices. In: Qurashi A, editor. Metal chalcogenide nanostructures for renewable energy applications, Publishing; John Wiley and Sons 2015, p. 189-232.

[2] Wang W, S. Lin A, D. Phillips J. Intermediate-band photovoltaic solar cell based on ZnTe:O. Appl. Phys. Lett. 2009;95:011103.

[3] Tanaka T, Nishio M, Guo Q, Ogawa H. Fabrication of ZnTe Light-Emitting Diode by Al Thermal Diffusion through Surface Oxidation Layer. Jpn. J. Appl. Phys. 2008;47:840.

[4] Wu D, Jiang Y, Yao X, Chang Y, Zhang Y, Yu Y, Zhu Z, Zhang Y, Lan X, Zhong H. Construction of crossed heterojunctions from $\mathrm{p}-\mathrm{ZnTe}$ and $\mathrm{n}-\mathrm{CdSe}$ nanoribbons and their photoresponse properties. J. Mater. Chem. C 2014;2:6547. 
[5] Wang K, C. Rai S, Marmon J, Chen J, Yao K, Wozny S, Cao B, Yan Y, Zhang Y, Zhou W. Nearly lattice matched all wurtzite CdSe/ZnTe type II core-shell nanowires with epitaxial interfaces for photovoltaics. Nanoscale 2014;6:3679.

[6] Artioli A, Rueda-Fonseca P, Stepanov P, Bellet-Amalric E, Den Hertog M, Bougerol C, Genuist Y Donatini F, Andr'e R, Nogues G, Kheng K, Tatarenko S, Ferrand D, Cibert J. Optical properties of single ZnTe nanowires grown at low temperature. Appl. Phys. Lett. 2013;103: 222106.

[7] Wang BB, Zhu MK, Hu N, Li LJ. Raman scattering and photoluminescence of zinc telluride nanopowders at room temperature. Journal of Luminescence 2011;131: 2550.

[8] Wan Buyong, Hu Chenguo, Feng Bin, Xu Jing, Zhang Y, Tian Y. Optical properties of ZnTe nanorods synthesized via a facile low-temperature solvothermal route. Materials Science and Engineering B 2010;171:11.

[9] You-Wen Y, Liang L, Min Y, Yu-Cheng W, Ting X, Guang-Hai L. Electrochemical deposition and properties of ZnTe nanowires array. Chin. Phys. Lett 2007;24:2973.

[10] Li Z, Salfi J, De Souza C, Sun P, V. Nair S, E. Ruda H. Room temperature single nanowire ZnTe photoconductors grown by metal-organic chemical vapor deposition. Appl. Phys. Lett. 2010;97:063510.

[11] Kirmse H, Neumann W, Kret S, D 1 uzewski P, Janik E, Karczewski G, and Wojtowicz T. TEM characterization of VLS-grown ZnTe nanowires. phys. stat. sol. (c) 2008;5:3780.

[12] Huo HB, Dai L, Liu C, You LP, Yang WQ, Ma RM, Ran GZ, Qin GG. Electrical properties of Cu doped pZnTe nanowires. Nanotechnology 2006;17:5912.

[13] Li S, Jiang Y, Wu D, Wang B, Zhang Y, Li J, Liu X, Zhong H, Chen L, Jie J. Structure and electrical properties of p-type twin ZnTe nanowires. Appl Phys A 2011;102:469.

[14] Hoomi S, Yousefi R, Jamali-Sheini F, Saaedi A. Large-scale and facile fabrication of PbSe nanostructures.by selenization of a Pb sheet. Functional Materials Letters 2015;8:1550063. 
[15] Wanger CD, Riggs WM, Davis LE, Moulder JF, Muilenberg GE. Handbook of X-ray Photoelectron Spectroscopy, Perkin-Elmer Corp., Physical Electronics Division, Eden Prairie, Minnesota, USA 1979.

[16] Nishio M, Kai K, Saito K, Tanaka T, Guo Q. Effect of VI/II ratio upon hotoluminescence and electrical properties of phosphorus-doped ZnTe films grown by metalorganic vapor phase epitaxy. Thin Solid Films 2011;520:743.

[17] Chen C, Zheng J, Nguy K, Naab F, D. Phillips J. Distinguishing Optical Behavior of Oxygen States and Native Deep Level Emission in ZnTe. Journal of Electronic Materials 2014;43:879.

\section{Figure captions}

Figure 1. FESEM images with different magnification and EDX spectra of the ZnTe nanowires that were grown at (a-c) 345 , (d-f) 375 , (j-i) $405^{\circ} \mathrm{C}$.

Figure 2. XRD patterns of the ZnTe nanowires that were grown in the different temperature.

Figure 3. (a) XPS spectrum of Zn and (b) XPS spectra of the Te.

Figure 4. PL spectra of the ZnTe nanowires. 


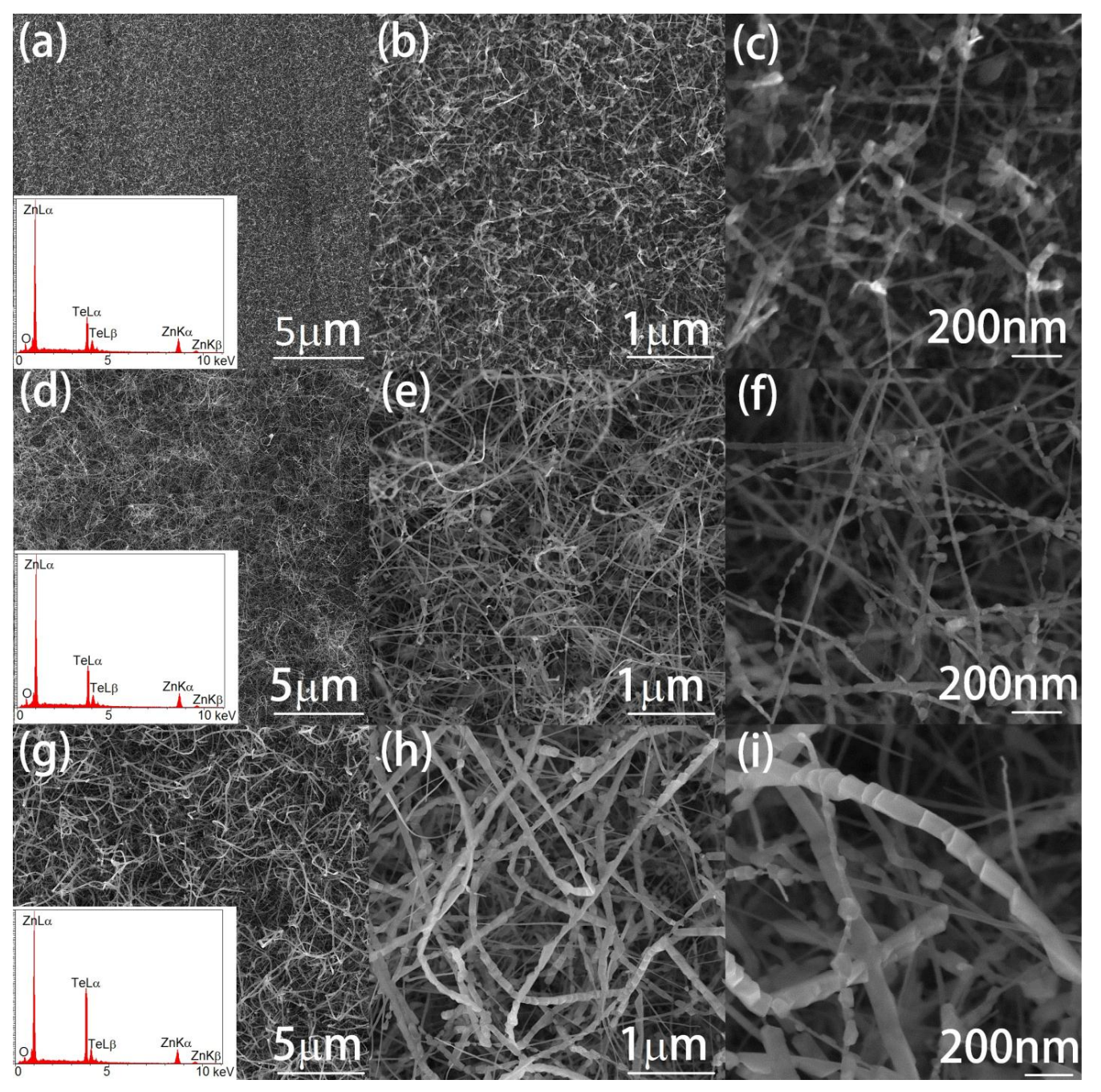

Fig. 1

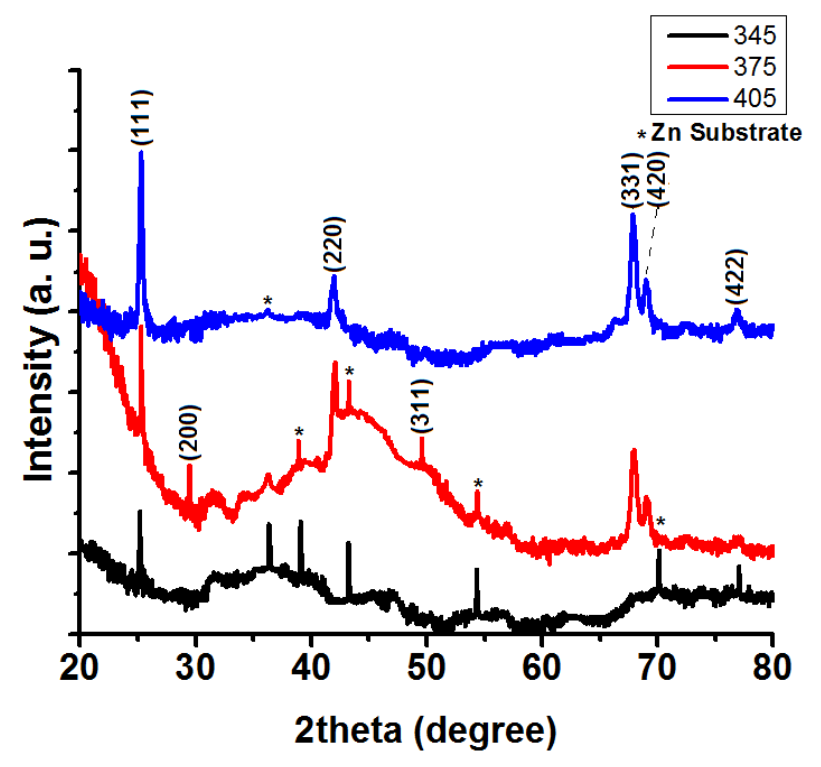


Fig. 2
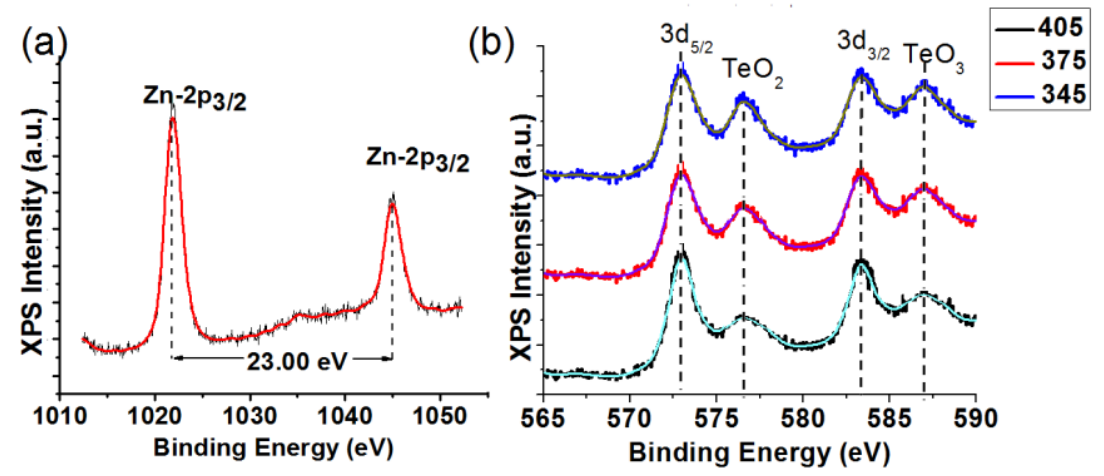

Fig. 3



Fig. 4 